\title{
DESCARTES. UN EJEMPLO DE CIENCIA NATURAL EN LOS ALBORES DE LA ÉPOCA MODERNA
}

I

El nuevo impulso cartesiano, que consciente y eficazmente trató de sustituir a la filosofía escolástica de las tres confesiones y cuyas huellas ostentan aún los sistemas por 300 años, no lo ocasionaron, con alguna verosimilitud, en primer lugar disquisiciones "filosóficas" (si se toma como base el fragmentario concepto actual de filosofía), sino el deseo de dominar la naturaleza. Por su parte, el deseo de dominar la naturaleza en este caso no ha brotado, con alguna verosimilitud, del desbordante sentimiento de dignidad humana, sino de la simple necesidad y de la conciencia de una aniquilación amenazadora. Tal deseo se apoya sobre una de las últimas oportunidades: el no restablecimiento ni la consolidación de las relaciones dominantes antes de la catástrofe, sino fuga hacia adelante. La catástrofe que mueve a huir hacia adelante, es la guerra confesional, que entonces se acostumbraba interpretar como la escalación de las disputas filosóficas de la Escuela; las relaciones económicas y sanitarias apenas si pueden soportarse, y la sociedad se encuentra en un callejón sin salida, del cual puede uno salir, como cree un puñado de hombres, únicamente sirviéndose de mejores modelos ideológicos. Tan pronto como los Estados ordenan éstos unitariamente, desaparece el motivo para la guerra ideológica; sin embargo, han de estar constituidos de tal manera que sean idóneos, como teorías conductoras, para una técnica y una medicina capaces de rendimiento. La paz se gana haciendo que puedan resolverse materias disputables y mediante la demostración de la existencia de Dios, que funda la moral, y de la inmortalidad del alma; con una teoría de la técnica, gracias a la visión de que el Universo es un continuo de corpúsculos, y de que estos corpúsculos sẹ mueven conforme a leyes matemáticamente representables; y, finalmente, con la teoría de la medicina, gracias a la demostración de que los cuerpos vivos son máquinas que se regulan a sí mismas, formadas por corpúsculos, y que no están sometidas a otras leyes que a las leyes naturales generales del Universo. Una teoría que puede rendir tanto, provoca el fin de las Escolásticas, que tan sólo concurren consigo mismas y de las cuales, según una extendida convicción, surgieron las guerras civiles confesionales, el fin de su explicación cualitativa de la naturaleza y, para concluir, el fin del status especial de los organismos. El dèseo de dominar la naturaleza apunta al alivio del hombre caído en apuros y exige una nueva ciencia. Ciertamente está ahí la ciencia, que subsiste ya desde Aristóteles, y 
se ha acreditado a través de los siglos; ¿por qué no se la quiere mejorar, sino sustituir?

Esta pregunta es difícil de contestar, porque habia métodos que hacian posible sostener en el marco de la Escolástica doctrinas completamente nuevas; ésta no era ningún sistema rígido, sino que se había mostrado inusitadamente flexible y capaz de adaptación. Así, el siglo xiv trajo una revolución de la dinámica, la teoría del ímpetu, de la que más tarde partió Galileo y que no podía encontrarse ni en Aristóteles ni en Tomás de Aquino; solamente la había sostenido Juan Filópono. A pesar de esto se impuso entre los escolásticos esa innovación, porque explicaba mejor el "movimiento violento" y porque en París les resultó bien a sus autores respaldarla con vagos pasajes de Aristóteles; ya en el siglo xv se tenía en general la opinión de que la teoría del ímpetu era la teoría dinámica de Aristóteles. Además, un canonicus de nombre Copérnico desarrolló un sistema no tolemaico del Mundo; en el siglo xvi, en la Universidad de Salamanca, de tendencia absolutamente tradicional, y que era pontificia, fue sostenido aquél como una hipótesis verosimil. Un tercer ejemplo: Tycho Brahe desarrolló un sistema helio-geocéntrico y el comentario aristotélico de los jesuitas de Coímbra, que en el siglo xviı era uno de los más conspicuos libros de texto en toda Europa, lo adoptó como hipótesis más verosímil. El contenido de una tesis, por tanto, tiene que haber desempeñado en esta época un papel secundario para su recepción; teóricamente habría sido posible que la filosofía escolástica admitiera también la teoría corpuscular o la atomista, toda vez que, entre tanto, había advertido cómo su física del continuo no explicaba particularmente bien fenómenos como la condensación y rarefacción de la materia. Asi se plantea de hecho la pregunta de por qué esta nueva física tan practicable, si el contenido de una tesis no era realmente lo decisivo, no fue asimilada inmediatamente por las Escuelas. La respuesta podría ser que sus autores se rehusaban a llenar el requisito para una admisión semejante.

La filosofía escolástica descansaba en autoridades; se remitía, siempre que le era posible, a autores reconocidos, a una reserva delimitada de bienes tradicionales; cuando lograba demostrar la compatibilidad de una nueva tesis con esos bienes tradicionales, no había ya ningún obstáculo para su recepción en el tesoro del saber por trasmitir. La demostración de la compatibilidad debía realizarse mediante la interpretación de las autoridades apropiadas -de manera semejante a como, en determinadas circunstancias, hoy se hacen sostenibles tesis científicas mediante comprobación con citas de Marx o de Lenin. En esta forma la interpretación vino a ser el instrumento más importante de la evolución del saber en la sociedad tradicionalista. La filosofía escolástica era un sistema de legitimidad tradicional; era legítimo lo que se combinaba con la tradición, es decir, que no la contradecía o, si la contradecía, se lo colocaba ahí junto, renunciando a competencia, en primer 
lugar como simple posibilidad ideológica, mientras la sociedad olvidaba su novedad (esto no solía durar mucho). En el caso de la doctrina del ímpetu, en Salamanca en el caso de la teoría copernicana y en Coímbra en el caso de la tesis tychoniana, tuvo éxito semejante admisión; con respecto a la teoría corpuscular y a la atomista, manifiestamente no lo tuvo. Pero, era natural que la Escolástica tratara en general de legitimarse con la tradición, pues el Occidente latino no desarrolló desde sí la llamada filosofía occidental, sino que la recibió de los árabes, quienes hasta el apogeo de la Edad Media administraron la herencia de la Antigüedad tardía y culturalmente eran superiores a los cristianos latinos; éstos recibieron su filosofía como una revelación, que sólo a través de generaciones pudo ser asimilada y reelaborada. La legitimidad tradicional se adapta a una situación semejante; garantiza el fundus heredado y protege a la sociedad de recaidas en el estado anterior a la recepción; debido a la posibilidad de asimilar nuevos bienes, el sistema no es solamente estable, sino al mismo tiempo flexible. Sólo se presentará una crisis cuando el tempo de la asimilación no sea suficiente o cuando las esperanzas y necesidades de la sociedad se desplacen de tal manera que la adaptación de los nuevos bienes exija demasiadas interpretaciones intermedias.

Si en el siglo xvir se quería mantener el status, había que demostrar mediante una interpretación la compaginabilidad de las nuevas tesis con las autoridades heredadas; esto habría sido una especie de testimonio de respeto de los innovadores hacia la tradición, que al mismo tiempo contenía una explicación de la renuncia a la agresión. Semejante testimonio de respeto tiene que ser experimentado como conveniente, mientras no se dé ninguna alternativa deseable respecto del status quo; el aferrarse a la tradición, a pesar de la indulgencia en los detalles, ha ofrecido usualmente, como la experiencia había enseñado hasta ahora, la mejor oportunidad para todos los partidos. Ahora bien, pareció llegado el instante en que con gran verosimilitud empezó a valer algo distiǹto; ésta es la razón de que hombres como Galileo y Descartes simplemente no respetaran la concepción tradicional de la ciencia de entonces. Lo único que se exigio de ellos fue que no atacaran activamente a la tradición, y no les habría sucedido nada, si hubieran legitimado sus tesis con citas de Aristóteles o de San Agustín. Descartes tenía amigos que buscaron para él citas adecuadas; sin embargo, renunció conscientemente a emplearlas. En ello consiste, tal vez, lo propiamente nuevo - no en el contenido de esta o aquella tesis, sino en algo meramente formal: el acto de la referencia a la tradición parece ser innecesario, más aún, se lo entiende como nocivo. Tiene que darse una alternativa, a saber, el conocimiento técnico del individuo informado; alguien se rehúsa a legitimar una tesis mediante la tradición, porque ha llegado a persuadirse de que. en .cuestiones de ciencia, si ha de evitarse la petrificación de errores, sólo es lícito 
dar una instancia legitimadora: la capacidad de juicio del especialista, adiestrada en la experiencia. Esta convicción permite una flexibilidad muy superior; ésta es la verdadera revolución, una ruptura de la concepción científica, el salto de la legitimidad tradicional a la legitimidad por comprensión. La posibilidad de un mejoramiento de la filosofía escolástica mediante adopción de teorías corpusculares habría persistido, pero no se recurrió a ella -no tanto porque la filosofía escolástica se negara a la recepción, sino porque los nuevos hombres se opusieron a que la tradición las recibiera, en vez de confesar su incompetencia básica. Su problema era liberarse de los contenidos de la tradición; ellos escogieron el recurso de la negación por principio y provocaron de ese modo el contragolpe de los conservadores de la tradición. El ataque de Descartes fue por lo demás muy indirecto; pues comprobó que al menos puede dudarse de las afirmaciones de la tradición, e introdujo en la ciencia la regla de que no es lícito hablar sobre algo dudoso.

Quien vive en un sistema de legitimidad tradicional, presupone que todo lo importante ya está contenido en la tradición; que, por decirlo así, San Agustín, Aristóteles y Santo Tomás escribieron de una vez por todas la verdad en sus libros. No puede creer que sea aún posible descubrir verdades en una extensión comparable; pero tampoco cree que sea necesario. En el siglo xvi, la opinión de que ya no se encontrarían muchas cosas nuevas era común y corriente; la mentalidad que le servía de base y cuyas huellas se encuentran también en textos de la primera mitad del siglo xviI, no facilita el trabajo de un innovador, pues de antemano hace aparecer inútil su iniciativa. También la tradición filosófica estaba fácticamente mezclada con la tradición teológica, pues los teólogos habían traducido sus afirmaciones al lenguaje de la tradición filosófica; quien ataque a ésta, ataca a la vez el lenguaje de los teólogos, y no es fácil decidir en cada caso particular dónde termina una disputa en torno a formulaciones y dónde empieza una disputa en torno a cosas. Las confesiones hicieron uso de todos sus medios en esta disputa y al final fracasaron. La tradición había sido conservada durante generaciones $y$, como todo lo conservado, era un factor de estabilización; al mismo tiempo, la homogeneidad religiosa de la sociedad regía como factor estabilizante; por ambas razones las autoridades se inclinaban asimismo a.luchar contra la nueva ciencia. También estas empresas fracasaron; sin embargo, relativamente pronto se les ocurrió a los políticos algo nuevo: el Estado liberal.

¿Qué clase de filosofía tradicional era aquella, a la que se enfrentaban los innovadores? Hasta entrado el siglo xviI se puede traducir la palabra "filosofía" casi siempre con la de "ciencia", porque su objeto comprendía también el material de lo que hoy se llama "ciencia". La primera disciplina 
de la ciencia es la llamada lógica o dialéctica, la doctrina de los conceptos, las proposiciones y los silogismos. Junto a ella está la gramática, como arte del manejo de símbolos lingüísticos para conceptos, proposiciones y silogismos, y finalmente la retórica como el arte de persuadir con ayuda de tales símbolos. Estas disciplinas forman el llamado trivium; es el vestíbulo de la filosofía en el viejo sentido, la cual se divide en materias teóricas y prácticas. En lo que se refiere a materias prácticas, existen desde Aristóteles tres: ética, política y economía. Las disciplinas teóricas se distribuyen en física y metafísica. Aún la física es una parte de la filosofía en el viejo sentido; todavía Newton llama a su disciplina natural y experimental philosophy; Kant empieza su carrera como escritor de filosofía con tratados sobre energía cinética y astronomía; y aún en nuestro siglo la física se cultivó en facultades de filosofía. Metafísica es, de acuerdo con una distinción que probablemente resulta difícil de concebir, la doctrina del ser, en tanto permanece inmóvil; física es la doctrina del ser, en tanto es movido o perecedero. Física en el viejo sentido, por consiguiente, es la doctrina de las cosas animadas e inanimadas de la naturaleza - de un lado, mineralogía, química, geología, meteorología, astronomía y semejantes, y del otro lado, en oposición al uso actual del lenguaje, como doctrina de las cosas vivas, al mismo tiempo botánica, zoología, fisiología y psicología. El lenguaje conserva recuerdos de ello: los médicos presentan un Physicum, en el cual se les examina no sólo sobre física, sino también sobre botánica y zoología; en los países anglosajones un hombre que no cultiva en absoluto la física en el sentido actual, es llamado physician, y no hace mucho tiempo se lo llamó entre nosotros todavía physicus. Junto a la doctrina de las cosas animadas e inanimadas, sin embargo, pertenecen aún a la física en el viejo sentido algunas disciplinas especiales, a saber: la perspectiva o dióptrica, la música o doctrina de la armonía acústica, una parte de la astronomía y la mecánica. Este grupo de disciplinas de la antigua física escolástica posee, a diferencia de las otras dos, la propiedad de ser matematizada; la óptica, la mecánica y la astronomía, en efecto, son desarrolladas mediante aplicación de símbolos geométricos, como secciones cónicas, radios y puntos; la música, en cambio, es desarrollada mediante aplicación de procedimientos aritméticos, como un sistema de proporciones numéricas.

La disciplina matematizada, decisiva para la ruptura ocurrida en el siglo xviI, es la mecánica. Ya en la Antigüedad fueron descritos procesos mecánïcos con la geometría y la aritmética; en aquel tiempo la mecánica consistía tan sólo en una estática relativamente completa. La Edad Media da un paso decisivo y desarrolla, como producto secundario de una doctrina general de las cualidades, la cinemática, pues el movimiento en el espacio era considerado entonces como una cualidad, entre otras cosas. Unos monjes de Oxford, los llamados calculatores, inventan al comienzo del siglo xIV un 
método para el cálculo de cualidades de toda especie según fórmulas generales, cuya forma especial para la aplicación al movimiento en el espacio todavía hoy se presenta como entonces: $s=c \cdot t, v=b \cdot t, s=1 / 2 b t^{2}$; pues " $\mathrm{s}$ " está por spatium, "c" por celeritas, " $\mathrm{v}$ " por velocitas y " $\mathrm{t}$ " por tempus; como en relación con la última fórmula la Edad Media hablaba de motus uniformiter difformis, aun hoy se dice "movimiento uniformemente acelerado", si bien la expresión "movimiento proporcionalmente acelerado" debería estar más cerca. La única fórmula que hubo de esperar hasta Beeckman y Galileo es: $s=1 / 2 g t^{2}$ para la caída libre; ella sólo podrá ser encontrada cuando la física sea ya tan especulativa como en el siglo xvi y no sea ya, como en el siglo XIv o Xv, una mera físiça de la percepción sensible; pues el máximo obstáculo para la aceptación de una fórmula general de la caída lo constituye por cierto la experiencia ingenua de que una pluma o una hoja caen mucho más lentamente que una piedra. Hacia 1320 unos escolásticos parisienses lograron la transposición de las fórmulas de Oxford a la geometría. Ellos desarrollaron un primitivo sistema de coordenadas, que consistía en un eje positivo de tiempo y un eje positivo de intensidad; si se inscribian en este sistema los valores de tiempo y de grado, resultaban figuras geométricas, que por cierto aún no eran desplazables dentro de su sistema de coordenadas, como más tarde en la geometría analítica, desarrollada en conexión con este principio. Una cualidad de intensidad constante produce un rectángulo; una cualidad uniformemente creciente, de intensidad y de tiempo cero, produce un triángulo rectángulo, cuya hipotenusa se eleva desde la base; una cualidad uniformemente creciente, de una intensidad mayor que cero produce un trapecio; y una cualidad variablemente creciente o decreciente, produce una cosa cualquiera, que en contraste con los triángulos, rectángulos o trapecios, no se podía calcular bien entonces. A semejantes figuras se las llama configurationes qualitatum, configuraciones cualitativas, y se las aplica a las más diversas cualidades como fiebre, fidelidad - gracia santificante; se cree tener en ellas la clave última para la esencia de las cualidades, y se piensa en amplias consecuencias para la medicina y la alquimia; tal parece como si entonces la física de los elementos hubiera alcanzado también algo que anteriormente sólo lograron los filósofos corpusculares: la explicación de efectos de las cualidades por medio de movimiento y figura. Así, contra las esperanzas de la Antigüedad, habían sido descubiertas también en lo cambiante estructuras matemáticas exactas. La doctrina de las configuraciones no pudo por cierto lograr lo que hombres como Oresme esperaban de ella; pero lo que sí permaneció, fueron las configuraciones cinemáticas, las configuraciones del movimiento uniforme y del uniformemente acelerado, las cuales, para concluir, cayeron en las debidas manos con- Galileo y Beeckman, tal como las había desarrollado el siglo xrv. Ellas representan probablemente el mayor logro de la Escolástica, nacidas en el tiempo 
de su supuesta decadencia. Ahora se sabe algo que la Antigüedad no consideró posible: no sólo los movimientos del cielo estrellado, sino también los movimientos espaciales de los cuerpos perecederos debajo de la luna obedecen a leyes matemáticas exactas.

Las disciplinas matematizadas de la física antigua constituian un grupo cerrado junto con las no matematizadas, como la biologia o la alquimia. Esta separación no era arbitraria, sino que respondía a las convicciones de la época. Asi, la doctrina de lo viviente no pudo ser presa de la matematización, porque el principio activo en sus objetos, los cuerpos y receptáculos, no era considerado como algo de que puede disponerse y medir, sino como algo anímico. Se admitió como dato fundamental una materia en su origen indiferente; en ésta es infundido (por las estrellas, por el alma del mundo o también por la naturaleza) un principio activo y en cierto modo anímico, que se designó forma sustancial; esta forma estructura la materia indiferente de acuerdo con su imagen, procura mantenerse en ella y actúa por su medio. Así, la forma (podría decirse: el alma) del agua es infundida en la materia indiferente y la configura en agua; sus propiedades características son peso y frío; por ello la ligereza y el calor son la negación del agua. El enemigo del agua tiene que ser el fuego, pues el fuego es ligero y caliente. Si la forma del fuego lucha con la forma del agua, es decir, si, -por ejemplo, se coloca una vasija con agua sobre la estufa, el fuego afecta con su propiedad activa, el calor, al frío y peso naturales del agua, hasta que la forma del agua no se puede mantener ya por debilidad y escapa de la materia poseida por ella; en su lugar avanza la forma del aire, que debido a su ligereza es similar al fuego (hoy diríamos nosotros: el agua se transforma en vapor, la materia que antes era fluida, se ha hecho gaseosa). Esta explicación se puede transportar a lo mayor. Si en invierno se mete la mano en una fuente, ésta produce an efecto relativamente cálido; si, por el contrario, se lo hace en verano, produce un efecto relativamente frio. Pues en invierno la forma del agua está segura contra su enemigo el fuego y no necesita afirmarse, sino que, por decirlo así, se puede relajar. En verano, por el contrario, cuando el sol abrasa, necesita hacerse especialmente fría, si es que quiere resistir el ataque. Un etnólogo podría confirmar que esto es un grado de racionalización bastante elevado; con una física de esta especie se pueden también explicar en forma plausible una cantidad extraordinaria de fenómenos; sin embargo, tiene la desventaja de que difícilmente puede matematizarse, pues sus formas sustanciales son una especie de almas, que no se pueden medir ni calcular. La Edad Media puede medir pesos, tiempos, superficies, extensiones y cuerpos, y la física tendrá oportunidad de ser matematizada sólo cuando renuncie a explicar los procesos naturales con la acción de formas no mensurables, y cuando intente describir la naturaleza con categorías cuyos objetos se puedan medir. Para este intento se tienen a la disposición las 
categorías aludidas; sin embargo, movimiento y figura son a la vez categorías de la estática y de la cinemática, y por ello el término: "matematización de la física", es sinónimo del término empleado por Dijksterhuis: "mecanización de la imagen del mundo".

Los dibujos de Isaac Beeckman, de Middelburg, muestran que Descartes se consideró ya a los 22 años como physico-mathematicus; y vio que su tarea era llevar las disciplinas de la ciencia todavía no matematizadas al nivel matemático de la estática y de la cinemática. Se sabe por qué le interesaba esto: quien pueda calcular la naturaleza, podrá dominarla; podrá, como dice el famoso pasaje del Discours, ser Maître \& possesseur de la Nature. Podrá aprovechar las fuentes de energía naturales, hacer máquinas para la agricultura y el transporte de carga, desecar pantanos y construir tiros de chimenea libres de humo, pero también podrá dirigir los organismos, determinar su crecimiento y la duración de su vida - no por obra de magia (esto lo habían pretendido muchos, y en ese tiempo no habría sido nada original), sino mediante ciencia controlable y asimilable para cada uno. Descartes creyó que todos los procesos de la naturaleza pueden describirse matemáticamente en forma total y que se hallan sometidos a leyes formulables matemáticamente; esta convicción implica un programa poco realista, que en el ínterin no se mostró ni como ejecutable ni como requerido o deseable; sin embargo, probablemente la grandeza histórica de esta física, que según los detalles ya no era para Leibniz otra cosa que ce beau roman, se halle precisamente en su irrealista programa: Descartes pensó el concepto de ley natural como una regla matemáticamente formulable, que puede verificarse en cada caso particular y gobierna sin excepción todas las regiones de la naturaleza, y no sólo en una visión general o para un dominio parcial determinado; él reivindicó los derechos de la música y la dióptrica, de la esgrima, meteorología, cosmología, fisiología, psicología empírica y embriología. En tal sentido esta empresa fue un paso históricamente necesario, por más que su fracaso se haya mostrado después tan claramente en todos los detalles; pues grabó en la mente de los contemporáneos la trascendencia del concepto de ley natural y la pretensión universal del nuevo tipo de ciencia y así allanó el camino para los trabajos de Huyghens y de Leibniz, quizá también para mucho de lo que ocurrió más tarde en el núcleo de la Royal Society.

Esta ciencia se caracteriza por el hecho de que persigue un programa práctico; pues apunta a la estabilidad política, a la técnica y a una medicina mecanizada. Por razón de este programa Descartes identifica el espacio, la extensión y la materia; éstos forman la naturaleza, el escenario de todos los procesos. En la naturaleza los procesos pueden describirse totalmente por medio de figura y movimiento de corpúsculos: los rayos solares así como la presión atmosférica, el desarrollo orgánico así como el nacimiento de las estrellas fijas en el cielo. El organismo humano es un sistema puramente 
mecánico, formado por corpúsculos de determinada figura y movimiento; quien haya reconocido esto, obtiene la posibilidad de alargar su vida a su gusto; Descartes piensa para sí mismo en algo así como cuatrocientos años, y en el fondo supo algo exacto, como muestra la estadística de población. Los modi figura y movimiento tienen ya tras de sí, al adoptarlos Descartes, una larga historia; con ellos se puede producir en la geometría de Arquimedes toda figura geométrica y todo cuerpo. Si la construcción de una fisica universal se logra mediante la descripción de figura y movimiento de corpúsculos, entonces quedará mostrado que la geometría euclidiana y arquimédica era sólo la geometría de la extensión pensada, mientras que la nueva física es la de la extensión real, la geometría del universo. El universo está matemáticamente estructurado, su gramática es la geometría cartesiana; quien la domine, podrá dar a la naturaleza órdenes precisas.

La esperanza en esto va extraordinariamente lejos e incluye también la posibilidad de dirigir al hombre. Esta parece ser más complicada, porque segủn la tradición cristiana el hombre no es solamente un organismo; debido a este punto esencial del cristianismo se plantea un problema especial a una filosofia corpuscular cristiana, puesto que para cada cuerpo humano ha de admitirse un alma. Aun cuando realmente todo en la naturaleza es figura y movimiento, sin embargo, el alma los experimenta como un sistema de cualidades, como verde, húmedo, cálido, aromático, angustioso o solemne. Descartes tiene que explicar estas experiencias por el hecho de que nuestra alma está intimamente ligada con nuestra máquina, porque Dios acopló a determinados movimientos de los corpúsculos en el organismo, determinadas señales o sensaciones cualitativas en el alma; si se dio lo uno, aparecerá lo otro con seguridad. En este acoplamiento Dios parte de puntos de vista pragmáticos; las sensaciones cualitativas sirven únicamente para la orientación rápida y segura en el espacio biológico; pero no sirven, en contraste con la opinión de la filosofía escolástica, para darnos informe sobre la naturaleza: éste lo pueden dar sólo las percepciones de figura y movimiento debido a su estructura corpuscular -el punto de arranque para la distinción boyleana y lockeana entre cualidades primarias y secundarias, que resultó de tantas consecuencias para la filosofía. Pues respecto de ella se plantea inmediatamente la objeción clásica: si con lo azul, lo cálido y lo angustioso, me equivoco sobre la esencia de los cuerpos, ¿de dónde recibo yo entonces la certeza de que no lo hago asimismo con lo uniformemente movido y con lo cuadrado? A tales objeceiones Descartes sólo puede enfrentarse remitiendo a la veracidad de Dios; con todo, de antemano resulta obvio que ha de reservar, de buena o mala gana, un status especial a la figura y al movimiento: se necesita de ellos, si se quiere hacer una geometría del universo; y conforme al estado de la técnica de medir hacia 16̧o, sólo ellos se pueden medir, no el sonido ni la luz, más aún, ni siquiera las temperaturas. Ahora bien, según Descartes, 
la tarea más importante de la filosofía es averiguar exactamente, con cuáles movimientos del organismo humano están acopladas determinadas sensacio. nes; pues por este medio resulta factible el último paso hacia el dominio completo de la naturaleza, el dominio de los afectos del hombre. Si en el organismo los afectos están acoplados a movimientos del todo definidos, el conocer las leyes del acoplamiento nos permitirá el descoplamiento mediante shock, así como la producción de afectos contrarios, y con ello una educación y una moral fundadas cientificamente. La nueva ciencia matemática debe hacer posible, según la necesidad, la sustitución de cobardía por valentía, de apatía por agilidad espiritual y de inercia por iniciativa; también debe permitir la inversión de predilecciones e inclinaciones humanas de acuerdo con las necesidades de la conveniencia. Semejantes operaciones, que en el siglo xvı se sentían aún como una esperanza, están ya conscientemente implantadas en la primera hora de la nueva ciencia.

Poco antes de 1630 Descartes termina de pensar el concepto de una ley natural universal - según cree, para lograr la posibilidad de una nueva técnica y medicina, en realidad, para abrir el camino a Leibniz, Huyghens y Newton. Sin embargo, en ello tropieza con dificultades específicas, pues todavía no existe la dinámica; y la explicación de los conceptos de fuerza y energía exigirá aún varias generaciones. El siglo xvil no sólo tiene el trabajo de explicar de dónde viene el movimiento; sino que, debido a su nueva concepción de la naturaleza, ha de explicar más allá de esto, por qué aquél sigue leyes matemáticas. Para Descartes, puesto que quiere lograr esa explicación, el único atributo de la materia resulta la extensión; por sí misma ella no es capaz de un solo movimiento, pues no contiene las formas que la física peripatética le atribuye indebidamente como principios de su actividad, más aún, no puede contenerlas; de otro modo reinaría en la naturaleza la anarquía de innumerables agentes particulares, y la representación de una ley natural universal sería tan sólo posible bajo condiciones adicionales difícilmente sostenibles. Con todo, es fácil concebirla, si se presupone que en la naturaleza sólo hay un único agente, y por cierto uno inteligente, el cual produce y distribuye todos los movimientos según un plan calculado por él. Así pues, debe ser Dios el que dota de movimiento a la materia -y a decir verdad, porque es inmutable-; en una cantidad constante; el primer principio de la conservación en la historia de la ciencia moderna está fundado teológicamente. A consecuencia del movimiento añadido a la materia según las leyes divinas, la materia adquiere sus modi concretos de figura y movimiento: Dios produce el movimiento como rectilíneo, pero en el choque con los cuerpos sufre determinadas modificaciones, que dan figura a la materia; si el primer corpúsculo es movido en forma rectilínea, desplazará a todos los otros corpúsculos de su lugar por razón de la plenitud del espacio; ellos serán forzados a apartarse hacia adelante, pero también hacia un lado 
y hacia atrás; en eso, la materia cambia la figura y el movimiento originarios, hasta que por fin, con base en la sabiduría de las leyes del movimiento, alcanza la figura ordenada; sin embargo, aun esto es en ella pasividad, no acción - como tampoco es acción nuestra cuando la lluvia cae sobre nosotros. Aquí opera una representación arcaica de pasividad, que por lo demás es suficiente en este estadio de la física. Para dar una respuesta a las dos dificiles preguntas, Descartes intenta algo tan inesperado como una mecánica universal, que no tiene el concepto de fuerza y se ayuda únicamente con extensión, figura y movimiento; pero no puede téner el concepto de fuerza, precisamente porque no puede tener el concepto de formas activas, si es que pretende hacer plausible el orden de la naturaleza.

\section{III}

Una mecánica sin el concepto de fuerza, empero, es verosímilmente la traducción de una teoría religiosa al lenguaje de la física. Esto sería instructivo, pues muestra que explicaciones anteriores del mundo pueden no sólo ser desplazadas por una nueva, sino también introducidas en ella bajo determinadas condiciones. La teoría religiosa de que se habla es la doctrina sobre la impotencia de la naturaleza: de los minerales, las plantas, los animales y los hombres. La creencia en su incapacidad perteneció, en una forma muy general, hasta ya entrado el siglo xvil, al permanente repertorio de la filosofía, pues la doctrina del concursus divinus y de la conservatio divina, común a todas las confesiones, explica que las creaturas no pueden existir ni obrar por propia fuerza. Descartes participa de esta teoría; aun uno de los más famosos libros del siglo xviri, la Teodicea de Leibniz, la encuentra a tal grado evidente, que menciona por su propio nombre a los tres autores que, según sus informaciones, dudaron de ella. Pero al lado existe una versión mucho más radical, cuyos representantes desechan la doctrina convencional del concursus como impía, laxa y pagana, ni siquiera aceptan conceder cooperación a los agentes naturales y en su lugar afirman: Dios es, en el sentido más estricto, el único principio activo en la naturaleza; las llamadas causas naturales no son otra cosa que signos o causas aparentes. Es de suponer que esta doctrina se infiltró del Islam en el Occidente, y a decir verdad por un camino que podemos seguir; con todo, desempeñó entre nosotros un considerable papel por largo tiempo y tal vez en un momento muy importante para nues. tra civilización tomó la función clave. Se trata de una doctrina religiosa, que lleva grabada la experiencia del poder y de la majestad del Creador y de la nihilidad de lo terreno; aparece en el Islam como reacción de la mística contra los naturalia aristotélicos y a fines del siglo xI toma su forma tradicional en un escrito del sufí persa Al-Ghazali, conocido en la Edad Media con el nombre de Algazel y dicho escrito bajo el título de Destructio philosopho. 
rum. Dos generaciones más tarde un eminente jurista, el Corregidor Ibn Roschd de Córdoba, a quien el Occidente llama Averroes, redacta en España una refutación ampliamente citada, que la Edad Media latina llama Destructio destructionis. Al-Ghazali afirma que no se da ningún nexo causal entre individuos determinados; la saciedad no presupone necesariamente el comer, el quemarse no presupone el fuego, y de cualquiera de las llamadas causas puede en principio seguirse cualquiera de los llamados efectos, pues el enlace de ambos descansa únicamente en la libre decisión de Dios, de crear ambas cosas sucesivamente. Dios es la causa única en la naturaleza; Él podría determinar exactamente igual que en vez de la saciedad, fuera el hambre la consecuencia del comer, sólo que no lo quiere. La objeción del sentido común contra esta tesis dice: si se niega cualquier nexo causal y se deja todo a la libre voluntad del Creador, se declara imposible la planificación y la espera racional del futuro. Quien al marcharse deja en casa un libro, podría encontrar a su regreso, en lugar de éste, a un hermoso muchacho o una pieza de oro; también podría haberse transformado el libro en un caballo, que ensuciara luego su biblioteca. A esta objeción Al-Ghazali da una respuesta que al moderno lector le recuerda la función del belief en la filosofía causal de Hume: Dios nos infunde determinadas esperanzas, que corresponden a lo que se designa como "curso ordinario de las cosas"; pero, tan pronto como tiene a bien cambiar el "curso ordinario de la naturaleza", infunde esperanzas correlativamente modificadas, y por ello sigue siendo posible para el hombre algo que puede designarse como esperanza del futuro y planificación.

La Edad Media conoció bien a Averroes y bajo su dirección refutó la doctrina causal de Algazel, como puede leerse por ejemplo en el Segundo Libro de la Fisica de Alberto Magno, así como en el Segundo Libro de las Sentencias, en el Tercer Libro de la Summa contra Gentes y en la primera parte de la Summa Theologica de Santo Tomás de Aquino. Tan pronto como una doctrina aparece en autores muy utilizados, es trasmitida con ellos y permanece viva con ellos, siendo enteramente igual, que se la ataque o defienda en primer lugar. Gracias a Averroes y a su recepción por la Edad Media latina, afloran en el Occidente los argumentos de Algazel y permanecen en juego por siglos. El que llegaran a tener nueva actualidad primero en la escuela de Ockham, no es extraño, pues Ockham explica que el nexo entre causa y efecto no es conocible ni por necesidad del pensar, ni a priori, y por ello sólo es conocimiento empírico de la posición y la secuencia temporal, no siendo posible la demostración científica de un nexo a priori causal. Esta concepción es conocida hoy como tesis de la Cuarta Sección de la primera Enquiry de Hume y en el siglo xviI no influye precisamente en forma original; nueva es en Hume, sin embargo, su incorporación a una filosofía del belief. En Ockham queda abierto, si (prescindiendo de la falta del nexo a priori) las causas naturales ponen ellas mismas su acción, o si Dios es 
la única causa. Esto se modifica en los prestigiosos representantes de la escuela de Ockham, y por cierto en los Comentarios a las Sentencias del cardenal Pierre d'Ailly, de Cambrai, y del primer ordinario de teología en Tubinga, Gabriel Biel, de cuya escuela provino Martín Lutero. El cardenal de Cambrai es conocido hoy por otras razones: él fue en el Concilio de Constanza el teórico principal del llamado conciliarismo, quien ideó algo así como una monarquía constitucional; fue además el autor de un difundido libro de texto de geografía, que ochenta años más tarde modificó la concepción sobre los continentes de Cristóbal Colón.

La ocasión externa que movió a Ailly y a Biel al desarrollo de su doctrina causal, no opera hoy como motivo; lo que interesaba era la cuestión de si un sacramento es la causa real o sólo la condición necesaria del efecto de la gracia ligado a él. Tomás de Aquino se había pronunciado, al comienzo del Cuarto Libro de las Señtencias, en favor de que es causa real; pues una condición necesaria sería meramente una causa per accidens, y la proposición de la Escuela reza: De causis per accidens non est scientia; ahora bien, la doctrina de los sacramentos es desde luego una ciencia. Frente a esto Ailly y Biel se inclinaban a ver en el sacramento sólo una condición necesaria; por ello se lanzó la objeción de que tales sacramentos producen menos efecto que las causas naturales. La respuesta no económica de Biel y Ailly a esta objeción explica que la naturaleza es del todo impotente, y que en ella no hay causas ningunas, sino sólo condiciones necesarias, que inducen a Dios, la causa única, a obrar. En este caso la causalidad de los sacramentos no se diferencia de hecho con respecto a la causalidad de las causas naturales; aun el fuego no es causa real del calor, sino que Dios resolvió, en libertad, producir usualmente el calor sólo en presencia del fuego. Entre ambos no existe ningún nexo causal; a la sola presencia del uno surge como es usual el otro, con base en una decisión divina y por divino influjo. Dios se conduce en ello, opina Biel, como un rey que dispone que el poseedor de un denario de plomo provisto con el sello del rey debe recibir cien florines del banco real; ciertamente este denario, si se quiere hablar así, es la causa de la recepción de cien florines, pero no en virtud de su naturaleza plúmbea, sino en virtud de la voluntad real. Si Dios resolviera que la lluvia debe caer solamente a la pronunciación de la palabra tacha, entonces esta palabra sería causa de la lluvia en el mismo sentido en que ahora lo es la ruptura de las nubes; ahora bien, tales causas, opinan Biel y Ailly, se llaman usualmente "condición necesaria".

Esta teoría levantó revuelo; todavía en los compendios escolásticos de los siglos XVI y xviI es combatida, por ejemplo, en Fonseca, los Conimbricenses y Suárez. Por este camino llegó hasta los cartesianos franceses posteriores, quienes, como Gousset y Malebranche, se remiten expresamente a Biel y Ailly a fines del siglo xvir. Con ello se esboza el primer empalme de la trasmisión, 
la tradición religiosa, que da respuesta al asombro del ser humano sobrecogido por la omnipotencia de Dios, afirmando que en esta naturaleza inválida y decaída, no obstante, algo se mueve. Pero, como es usual, la respuesta no fue más lejos que la pregunta. Quien preguntaba no quería en modo alguno saber qué es lo que se mueve y de acuerdo con qué leyes, pues esto es decreto de Dios. No es incomprensible que ciertos hombres, en virtud de una experiencia religiosa determinada, o también en virtud de una especial tradición religiosa, puedan llegar a una cuestión semejante, que tiene consecuencias prácticas para su vida; mas, por otra parte, no es ésa la única pregunta que un hombre puede plantear con respecto a la naturaleza. Se dan situaciones diferentes a la del que reza, el cual no puede comprender que algo creado a su vez cree, por ejemplo, la situación del físico en el viejo sentido, que querría saber cómo llega la naturaleza a un orden tan ingenioso, o la del médico que querria saber por qué los organismos hacen frente de una manera tan asombrosa a las más diversas exigencias. El intento de dar una respuesta a esas preguntas lo hace otra tradición, que tuvo mucho éxito en la historia. Ésta pretende hacer comprensible que el maravilloso ordenamiento de la naturaleza no es incomprensible, y en ello se conecta probablemente con tradiciones estoicas. Según la tesis de la stoa la divinidad se infiltra en el Universo

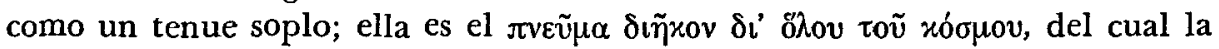

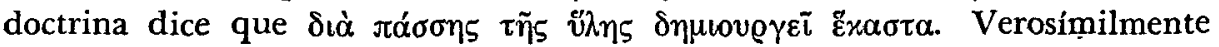
bajo el influjo de esta doctrina, que implica una afirmación causal, se originó en la Diatriba la representación de un espírtu protector y maestro de los organismos, la tutrix animalium, que en el Renacimiento representa un papel más considerable entre los médicos, pues por razón de su oficio éstos se ocupan de la complicación de los organismos. Así se dice en un passus de la $A n$ toniana Margarita, del médico Gómez Pereyra, de Medina del Campo, que apareció en 1554 y en la cual se introduce por primera vez en la fisiología la metáfora sobre los autómatas, que todos los movimientos animales y todos los procesos involuntarios vegetativos y sensitivos, delatan una sabiduría que supera no solamente a la capacidad de los organismos, sino también a la comprensión humana. 'De ahí que sólo pueden ser ideados por una causa enteramente sabia, que jamás se equivoca con respecto al todo y que dirige las facultades vegetativas y sensitivas de los organismos; esta causa se llama tutrix animalium. Sobre su naturaleza no se hace en la Antoniana ninguna afirmación definitiva: "Si es una primera causa, que está en todos los lugares, infatigada e infatigable, por muchas que sean las tareas que le incumben, entrelazadas unas con otras; o si es otra, la causa segunda, aquella inteligencia que asiste siempre en la superficie entera de la tierra y está siempre presente en todas sus partes, la que por cierto se llamó entre los antiguos filósofos alma del mundo, que dirige la formación de los embriones y los movimientos de los seres irracionales; o si a inteligencias diferentes incumben negocios tan dife- 
rentes: ninguna de esas proposiciones es absurda o no se ajusta a la verdad" (Col. 140).

Semejantes pasajes no traen ninguna afirmación general sobre causalidad; solamente explican que un principio sabio enseña a los organismos sus maravillosas reacciones y dirige sus movimientos hacia una meta ideada por una sabiduría superior; pero no se explica que el alma misma del mundo produce esos movimientos, o que los organismos no son los principios de sus propias actividades. Una afirmación semejante daría respuesta a una pregunta que en general no se planteó en esa situación de modo inmediato; uno se entera de que la naturaleza está en movimiento, mas queda en cuestión por qué no simplemente está en movimiento, sino que lo está dentro de ese orden tan asombroso. El Dios de Al-Ghazali o el Dios de Ailly y Biel hace lo que quiere; la respuesta de éstos no tiene como fin saber si lo que El hace es sabio conforme a nuestra inteligencia, sino saber que lo hace con entera libertad, en contraste con la débil criatura. En el principio inteligente de los médicos, en cambio, el acento se halla sobre que él actúa con sabiduría; y no está a debate, si, por lo demás, es también libre, o si los organismos son impotentes, sino el hecho de que en sus actividades aparece esa maravillosa armonía. Ciertamente es posible que estos dos motivos se fusionen uno con otro; así parece haber sucedido al principio del siglo xvir en la filosofía natural antiaristotélica del médico calvinista Sebastián Basson de Montbéliard: aquí, de acuerdo con un buen uso de los médicos, es introducida el alma del mundo y, al mismo tiempo, de acuerdo con un buen uso de los cristianos, se introduce la doctrina de la impotencia de las causas segundas, como apareció en Biel y Ailly; la antigua convicción religiosa de que las cosas no pueden obrar por sí, se enlaza en una unidad con la antigua convicción de la filosofía natural, de que ellas mismas no son capaces de idear su propio orden.

Sobre la relación de Basson con Ailly y Biel, por el momento sólo es posible hacer conjeturas; sin embargo, algunos datos hablan en favor de que él los conocía. Las citas de la Philosophia naturalis muestran que poseía un conocimiento desusadamente bueno de autores escolásticos tardios; también él introduce la doctrina con el término "presencia", característico de Biel y Ailly. En Basson encuentra uno ya el repertorio de argumentos, del cual se servirá treinta años más tarde la Physiologia Christiana: causalidad es exclusivamente un atributo de Dios, y por ello ha de desecharse la filosofía aristo télica, que asigna también causalidad a las cosas naturales, como idolatría, como pecado y locura (II De Forma, III 7). Delante de este trasfondo cristiano aparece la antigua "naturaleza" de los médicos: el Dios presente por doquiera produce todo inmediatamente (II 10), mueve los corpúsculos de acuerdo con la armonía, en que ellos componen la forma fluens de las cosas, y hace que de sus modificaciones se sigan las modificaciones de sus actividades (III De Forma, II 9). Esta causa divina mueve a las cosas de acuerdo con su ser cósico, 
así como un trabajador manual mueve su herramienta (I 1). De esta manera, la razón divina, derramada sobre todo, da a todas las cosas movimiento y fin; ella es el citaredo divino, quien arranca a las cuerdas, que él mismo tensó, agradables modulaciones y quien conserva la armonía así en lo individual de la naturaleza como en el conjunto. Este Dios incesantemente activo y presente en todas las cosas se llama natura naturans (I 5 ); el alma del mundo, que lo penetra todo, la cual también se llama "éter", forma con él una unidad funcional: ella es el instrumento en la mano de la naturaleza naturante (De Natura, III 3).

Esta doctrina aporta algo nuevo en cuanto que en ella las dos situaciones esbozadas están fundidas en una sola. El calvinista reconocía la magnificencia de Dios; sabe que Dios hace todo y que lo hace como El quiere; pero, al mismo tiempo, el médico sabe que, a pesar de todo el ocultamiento del decreto divino, el ojo del hombre puede reconocer la asombrosa armonía y el orden imponente de ese obrar divino. En este punto la antigua tesis religiosa perdió su hostilidad agustiniana contra la investigación y su anticuriosité; ahora podrá hacerse cargo de su función para la historia moderna y preparar el camino para algo nuevo, en donde ella misma ya no tiene ningún sitio. Ciertamente, también sobre la relación de Basson con Descartes sólo es posible hacer conjeturas; ambos autores se hallan cerca uno de otro objetiva y temporalmente, pero nadie sabe cuán bien conoció el autor de Le Monde la filosofía de Basson. Está confirmado que conoció su nombre y algunas de sus tesis cuando más tarde en 1630; pero no se puede determinar cuáles. Además es claro que la física de Basson se parece asombrosamente en algunos puntos a la física de Descartes: ambas desarrollan un sistema corpuscular, en ambas desempeña el éter funciones análogas, y ambas rechazan el espacio vacío con base en presupuestos muy semejantes. Con ello, por cierto, no se ha demostrado nada.

Para tener una respuesta a la pregunta sobre el origen y la distribución del movimiento según leyes, la física cartesiana, como se había mostrado arriba, hace dos presupuestos fundamentales: la naturaleza, según su esencia, es impotente, pues todo movimiento en ella fue producido por una causa externa, a saber, por Dios; y Dios mueve a la naturaleza según leyes infinitamente sabias y formulables matemáticamente, que el autor designa en forma asombrosa como causas secundas (Princ. Phil. II 37). Causa secunda es un término equívoco, que de ordinario significa simplemente "creatura"; existe, sin embargo, un significado especial —sostenido, por ejemplo, en Gómez Pereyra-, según el cual causa secunda significa una inteligencia creada, subordinada a Dios, que administra la naturaleza - algo así como el alma del mundo o como el espíritu protector de los animales. Ese significado especial ofrece el mejor sentido en conexión con la ley natural; pues, si estuviera a la vista, Descartes concluiría: las leyes naturales no son por cierto inteligencias o sustancias. 
espirituales, pero sí llenan exactamente las mismas funciones que físicas menos valiosas atribuyeron a semejantes inteligencias naturales hipotéticas; se desprende de varios pasajes (AT II 41 , XI $3^{6}$ y XI 524) que Descartes tiene por disparatada la admisión de tales inteligencias. Sin embargo, de todos modos parece admitir para el movimiento de la naturaleza un principio distribuidor inteligible, si bien no sustancial; y por ello, el hablar de causa secunda ofrece un cierto sentido, pues hace referencia a una analogía y señala al lector lo que se entiende aquí. La imagen de la naturaleza trazada por Descartes se adapta bien a ello; en la mecánica sin fuerza aparece la faz de la naturaleza impotente y administrada.

Así, verosímilmente hacia 1630 , esta doctrina tradicional viene a ser importante en un sentido ajeno a ella en sus orígenes; pues (distanciada de su fin y despojada de su función religiosa) se convirtió de manera imprevista en una de las condiciones previas de la civilización técnica, ya que explica así la producción como el curso legal del movimiento: lo uno lo ejecuta la causa prima; lo otro, la causa secunda legal-natural. Semejante construcción se hallaba empero extraordinariamente cercana a Descartes; a quien pretenda llevar la física entera al nivel matemático de la mecánica, antes de haberse inventado la dinámica, le será esto relativamente fácil, si admite que en la naturaleza no hay fuerzas ningunas, que Dios es la causa única del movimiento y que todos los movimientos de los cuerpos en la naturaleza se hallan sujetos a leyes matemáticas. Sin embargo, la hipótesis de que la trayectoria de la piedra que cae, es por ejemplo $1 / 2 \mathrm{gt}^{2}$, presupone algunas cosas, por más que nosotros usualmente damos sin titubear la respuesta de que obedece a la ley natural. ¿Acaso pueden obedecer los cuerpos? $O$ ¿puede una piedra hacerse el cálculo de la fórmula $1 / 2 \mathrm{gt}^{2}$ ? Si no: ¿puede alguien enseñársela, para que sepa dónde ha de detenerse al caer? Contra tales preguntas, que más tarde provocarán al idealismo trascendental, Descartes defiende su tesis sobre la total pasividad de la materia; ninguna piedra y ningún cuerpo pueden ser la causa de sus movimientos siempre racionales, pues ninguna piedra y ningún cuerpo es racional y puede pensar; los cuerpos no pueden contener el principio de su movimiento y, si la naturaleza debe permanecer ordenada y no resultar un caos, tienen que ser impotentes y estar movidos y dirigidos por un principio externo, por aquel matemático divino, que ahora, debido a una razón imperiosa, reúne la función de la causa universalis de Biel y Ailly con aquélla de la omnisciente alma del mundo de los médicos. Pero, con ello se ha alterado también algo en la representación de Dios; el "espíritu del mundo" no es ya sabio sólo a la manera de un hábil padre de familia; de aquí en adelante será el matemático sin más. Así, la tesis cartesiana sobre la producción y distribución del movimiento, tomada en abstracto, causa por cierto una impresión muy extraña; si, en cambio, se la contempla en el marco de su situación, se muestra como aquel aserto que, en el mundo dado de re- 
presentación filosófica, pudo conducir antes que todos a un contexto aceptado. La historia de la filosofía, por lo demás, ofrece al respecto algo así como una prueba en contrario. Leibniz introduce de nuevo - y no en último término como reacción a la física cartesiana- un concepto purificado de fuerza: La substance est un être capable d'action. Sólo que él afirma que toda sustancia posee fuerza y es capaz de obrar, porque al mismo tiempo está dispuesto a conceder que es también capaz de percibir; en ello reside todavía para él una consecuencia del todo evidente. Más adelante aún Kant presentará la propuesta de que la racionalidad de los movimientos de los cuerpos no encuentra su fundamentación ni en los cuerpos ni inmediatamente en Dios, sino en el sujeto trascendental; en el fondo el problema siguió siendo el mismo.

Que la física cartesiana incorpora en su contexto la doctrina tradicional sobre la impotencia de la naturaleza, es una mera conjetura; sin embargo, varias razones hablan en su favor. La doctrina de Al-Ghazali, Ailly y Biel estaba consignada en los libros de texto que usaron los maestros de Descartes, y que en parte Descartes mismo consultaba, con los nombres de sus autores; pues solía venir a discusión en el tratamiento de la doctrina causal así en la física como en la metafísica. Además, en dichos manuales se señalaban tesis de autores como Pereyra y Basson, usualmente sin mención del nombre; también se hallaban en la boca de los curieux. La doctrina sobre la anima mundi fue conocida por Descartes, pues polemiza contra ella; y, finalmente, su física muestra sugestivas analogías con las doctrinas mencionadas. Se puede comprobar en parte que supo algo de ellas; lo único que no se puede demostrar en forma estricta es que las haya tomado como modelo o contramodelo en el desarrollo de sus teorías. Pero si la conjetura mencionada fuera correcta, entonces la doctrina de la impotencia de las causas segundas tomaría a su cargo hacia 1630 , por dos razones diversas, la tarea de hacer posible la gran meditación cartesiana sobre la legàlidad de la naturaleza, pues, en primer lugar, hace aparecer normal una metafísica sin concepto de fuerza y, en segundo, da una razón plausible para la legalidad en los movimientos de los cuerpos. En ese contexto ya no desempeña primariamente su función religiosa ordinaria, de presentar a los creyentes la omnipotencia y majestad de Dios, sino la intramundana, de hacer concebible la naturaleza como un sistema dominado por leyes matemáticas.

Sea cual fuere lo que se diga de èsta conjetura - una considerable parte de la tradición cartesiana entendió las afirmaciones de Descartes en ese sentido. La física cartesiana había fracasado en poco tiempo; tan pronto como empezó a abrirse paso la voluntad de una dinámica como parte de la física y no de la teología, la representación de la impotencia de la naturaleza ofre. ció al físico oportunidades considerablemente menores que antes, resultó cientificamente poco interesante y retornó al arsenal de la religiosidad. Quien 
la sostuvo después de la muerte de Descartes, la sostuvo menos por un interés civilizador que por uno religioso, del cual habla surgido primero. Términos con larga tradición poseen poder; incluso cuando son despojados de significaciones dudosas y, bien definidos, deben cumplir sólo funciones auxiliares, no se puede decir siempre con seguridad, si una escuela los recibió en su mano o si ellos recibieron en su mano a esa escuela. La representación de la impotencia de la naturaleza no sólo recuperó hacia el fin del cartesianismo su dimensión religiosa originaria, sino que rehuyó además su función civilizadora. El movimiento cartesiano, cuyo fundamento meditativo, tal vez de acuerdo con un modelo ignaciano, debió ejercer una acción social y política, termina en la contemplación, ajena a los hechos, del poder y de la sabiduría de Dios y de la impotencia de toda creatura. Éste es el estado final de la Escuela, como se lo alcanzó en los trabajos casi hostiles a la ciencia de Geulincx y Malebranche. Para la historia de la espiritualidad europeo-occidental significa una importante culminación, para la historia de la ciencia, un retroceso; este conflicto podría ser sintomático. A pesar de esto, Malebranche celebra al principio del siglo xviI una asombrosa resurrección en la filosofía de George Berkeley, quizá el único filósofo británico después de Locke, que puede ser entendido con la mayor facilidad desde el Continente. George Berkeley es el último sustentador filosóficamente grande de la doctrina de la impotencia, y si su filosofía natural, que es más reciente que la física newtoniana, no hubiera sido anacrónica, habría aportado a la humanidad un considerable beneficio y habría librado a los físicos del lastre de la dinámica y a los matemáticos del lastre de lo infinitesimal. La nostalgia berkeleyana y malebrancheana por el religioso recogimiento de la prerrevolución científica es, por lo demás, ambivalente, pues la revolución cientista de la temprana época moderna, que aparece a los teólogos como ruptura de la tradición, salvaguardó al menos en un aspecto los intereses del cristianismo: la sociedad había llegado a un punto en que el mandamiento central del amor no podía ya cumplirse con medios tradicionales como obras de caridad y atención de hospitales, sino tan sólo con una técnica y medicina que funcionaran bien sobre la base de una ciencia segura. Cuando esta posibilidad tuvo que ser realizada en contra de la oposición de los teólogos, los custodios de la religión cristiana pusieron en peligro, por extraña coincidencia, el cumplimiento del mandato capital del cristianismo. Así pues, luego que la representación de la impotencia de las causas segundas hubo caracterizado por un instante el futuro de Europa, apenas unos años después autores como Geulincx, Malebranche y Berkeley se apoyaron precisamente en ella para volver a la oración y reverencia "precientíficas" con su desesperación civilizadora. 
IV

El intento de Descartes de dar una respuesta a la pregunta por la proceden: cia y por el curso legal del movimiento con. su tesis sobre la pasividad del hombre, llevó a un contexto que tuvo extraordinario exito y durante una serie de años se mostró consistente; sólo después de más de una generación fue puesto en duda fundamentalmente por el desarrollo de la mecánica clásica. Sin embargo, en el curso de la ulterior reflexión se hizo claro que deparó a la ciencia una cantidad de nuevos problemas; esto fue el origen de su fecundidad. Sobre todo, el desarrollo de una fisiología mecanicista en el marco de la física cartesiana exigió como contraste la llamada separación de cuerpo y alma; encontrar para ésta una versión creíble fue extraordinariamente difícil, como lo hicieron ver los intentos de los partidarios del influjo y de los ocasionalistas, asi como las contraofertas de Spinoza, Leibniz y Kant. La psicofísica siguió siendo por décadas el hilo conductor del desarrollo sistemático en el Continente y aquí proporcionó los motivos más convincentes para una reforma de la nueva física, mediante incorporación de los conceptos de energía y dirección.

Mas el intento cartesiano produce efecto aun por encima del marco de la ciencia y conduce a una crisis general, cuyo aspecto religioso es tal vez aún el menos importante. La legitimidad tradicional era la forma de constitución de la teología revelada, y quien la combatiera en la ciencia, como Descartes, provocaba la solidaridad constitucional de los teólogos. Los teólogos tenían razón en el fondo, pues a pesar de la gran hostilidad contra su política ciertamente la crítica de los innovadores excluyó al principio el dominio de la revelación, mas pronto se mostró lo provisional de una conducta semejante; ya en los años setenta la revelación se había convertido en objeto de crítica en los trabajos de Spinoza y Simon, pasando por Hobbes. Pero en tanto que la teología se concibió como interpretación sistemática de la revelación, habia llevado a cabo una autosintesis con la física aristotélica; sus dogmas estaban formulados en el lenguaje de esta física, y reaccionaban al ataque contra ese lenguaje con violencia $y$, por ello, una vez fracasados, permanecieron hasta hoy como sospechosos. Habrá de concederse que era difícil encontrar una alternativa deseable; por ejemplo, si repentinamente los dogmas religiosos se hubieran formulado en lenguaje cartesiano, cincuenta años más tarde se habría llegado nuevamente a la misma perplejidad, cuando ya el cartesianismo hubiera sido superado. Como, por lo demás, los teólogos de una religión revelada tienen ideas precisas de la verdad, caen en especiales dificultades con el pensamiento de que lo principal de la ciencia no es "la conservación de la verdad", sino el hallazgo de explicaciones afortunadas - un punto de vista, que en otros círculos fue expresado ya en el siglo xvir. La hostilidad de los teólogos impone a los innovadores la tarea de ser, por decirlo así, como 
las confesiones, religiosos o cristianos; o de ser, comó las religiones, buenos y nobles; el punto de vista de que los teólogos se oponen con su lucha al fenó meno que más tarde se llamará progreso, conduce a una paulatina descristianización de la humanidad.

El segundo aspecto de la crisis es la inseguridad de la relación hacia la tradición, que aun políticamente se muestra plena de consecuencias, y con la cual se enfrenta todavía después de ciento cincuenta años la teoría política de la restauración. A ese respecto es extraño cuánto les falta a los innovadores la visión de que ellos mismos permanecen atados, en su terminología, sus modelos ideológicos y sus puntos esenciales, a la tradición en una medida asombrosa; su autoexposición prescinde de ello de manera tan convincente, que sólo en nuestro siglo se ha delimitado la proporción de su tradicionalidad en forma aproximativa. Su nueva ciencia únicamente reconoce como instancia decisiva la perspicacia del especialista; esto conduce muy pronto al distanciamiento aun de las tradiciones sociales y estatales, y a la necesidad de un nuevo concepto ético. Una especie de reconciliación de esa perspicacia con la tradición la trae primero el llamado despertar del sentido histórico, que por principio remite a la razón humana a su finitud y no por eso la empuja precisamente a depresiones. Esto resulta comprensible, si se supone que en el siglo xviı parecía exigirse demasiado a la razón humana. Los grandes éxitos del nuevo tipo de racionalidad condujeron ya en Descartes a un cambio de la disposición de ánimo; ahora se está convencido de que para el hombre casi todo es posible, calculable y abarcable con la mirada. A esta convicción se le enfrentan a lo largo de unos ciento cincuenta años progresos sólo relativamente modestos e impresionantes fracasos, que hacen apa: recer comprensible el descontento con la razón, toda vez que ésta ha causado aquel extraño aislamiento, bajo el cual sufre ahora el hombre. No sólo su relación con las emociones, sino también su relación con la naturaleza que lo rodea, está sensiblemente perturbada, pues Descartes la declaró, en cierto modo de la noche a la mañana, impotente e inanimada, después de que ella era hasta hacía poco todavía la gran salvadora y madre de todos los seres. Esta nueva interpretación implica especialmente una crisis de la corporeidad con muchos aspectos; el amor corpóreo se revela, por ejemplo, como amor a las máquinas, y la procreación es reproducción de máquinạs bajo condiciones sobradamente extrañas; también se agudiza la conciencia de que la comunidad de los espíritus en el plano del hombre se lleva a efecto sólo mediante un sistema de señales sumamente complicado y no unívoco, y el problema de la comunicación se plantea con agudeza que antes apenas si existió. Finalmente surgen aún dificultades para reconocer de nuevo al padre en el matemático divino. El hombre, convertido en un ser sin madre y solitario emprende en lo sucesivo una serie de intentos de fuga del dominio de la razón, que se le ha hecho algo difícil de soportar, hacia la naturaleza, que no empieza 
primero en Rousseau y todavía hoy no ha llegado a un término duradero. En otro nivel se da la fuga hacia la historicidad, donde es proporcionada la naturaleza y se concede al individuo saberse admitido en el gran proceso total. Pero, finalmente, la crítica de la razón llega a través del "sentido histórico" al resultado conciliador, de que la autoapreciación de la razón en el siglo xvir descansaba en errores que, sin embargo, eran fructíferos. Como quiera que sea, el impulso cartesiano provocó etapas enteras de la historia posterior del pensamiento; fue demasiado bueno para no convencer a primera vista, y a la vez demasiado malo para resolver en forma duradera la mayor parte de los problemas paticulares planteados a él; tal vez yace ahí el secreto de una gran filosofía.

UNIVERSidad DE MANNheim

REINER SPEChT

[Trad. de Bernabé Navarro] 